\title{
VIDEOGAME E SUA INFLUÊNCIA EM TESTE DE ATENÇÃO
}

\author{
Luciana Alves \\ Alysson Massote Carvalho"
}

\begin{abstract}
RESUMO. Esta pesquisa objetivou investigar a performance em cinco variáveis do Continuous Performance Test (CPT-II), um teste de atenção sustentada. Dela participaram 30 indivíduos, com idades entre 10 e 16 anos, envolvidos nas atividades de uma organização não governamental (ONG) para crianças carentes, organizados em dois grupos: um grupo de jogadores de videogame (JVGs) e um de não jogadores de videogame (NJVGs). Todos eles foram testados no CPT-II e o grupo de NJVGs foi treinado por 20 sessões em dois jogos de videogame. Os NJVGs foram retestados no CPT-II. Verificou-se que, inicialmente, o grupo de JVGs apresentou um desempenho melhor do que o grupo de NJVGs. Após a intervenção experimental constatou-se que este último grupo apresentou uma melhor performance nas variáveis avaliadas do teste. Os resultados mostraram que o regime de treinamento e os jogos de videogame utilizados foram eficientes para melhorar o desempenho de adolescentes NJVGs no CPT II.
\end{abstract}

Palavras-chave: Videogame; atenção; Continuous Performance Test.

\section{VIDEOGAME IMPACT IN ATTENTION TEST}

\begin{abstract}
The present research aimed to investigate the performance in five variables of Continuous Performance Test, a maintained attention test. Participants included 30 individuals, aged between 10 and 16 years old, involved in the activities of a Non-governmental Organization (NGO) for poor children, organized in two groups: one of videogame players (VGP) and another with no-players videogame (NVGP). All of them were examined in the CPT-II and the group of NJVG was trained for 20 sessions in two videogames. The NJVG were re-examined in the CPT-II. It could be verified that the group of JVG showed a better performance than the group of NJVG before experimental intervention. After experimental intervention it could be noted that this last group presented a better performance in the test variables evaluated. The results showed that the training conduction and the videogames used were efficient for improving the NVGP adolescents' performance in the CPT-II.
\end{abstract}

Key words: Videogame; attention; Continuous Performance Test.

\section{VIDEOJUEGOS Y SU INFLUENCIA EN LA ATENCIÓN}

RESUMEN. Este estudio tuvo como objetivo investigar la actuación de cinco variables en Continuous Performance Test (CPT-II), una prueba de atención sostenida. Participaron 30 individuos con edades entre 10 y 16 anos, envueltos en las actividades de una Organización no Gubernamental (ONG) para niños carentes, organizados en dos grupos: uno para los jugadores de videojuegos (JV) y otros con no jugadores de videojuegos (NJV). Todos se pusieron a prueba en el CPT-II y el grupo de NJV se formó por 20 sesiones en dos videojuegos. El NJV fueron re-probado en la CPT-II. Se encontró que en principio, el grupo JV mostraron mejor rendimiento que el grupo de NJV. Después de la intervención ensayo encontró que este último grupo mostró un mejor desempeño en las variables de la prueba. Los resultados mostraron que el sistema de formación y los videojuegos utilizados son eficaces para mejorar el rendimiento de los adolescentes NJV en CPT II.

Palabras-clave: Videojuegos; atención; Continuous Performance Test.

A atenção envolve redes neurais especializadas que executam funções como manter o estado de alerta, orientando para eventos sensoriais, e controlar sentimentos e pensamentos (Posner \& Fan, 2006).
Independentemente do modelo teórico adotado para sua abordagem, a atenção inclui a sua sustentação por um determinado período de tempo, relacionada com o estado de alerta ou vigilância, além de inibição

Psicóloga. Mestre em Ciências da Saúde. Atua no setor de Avaliação de Tecnologias em Saúde da SES/MG.

\# Pós Doutor em Psicologia. Orientador do Programa de Pós-graduação em Ciências da Saúde - Saúde da Criança e do Adolescente - Faculdade de Medicina (UFMG). Atua como Diretor Geral do Instituto Presbiteriano Gammon. 
de estímulos irrelevantes, com consequente focalização nos estímulos de interesse, denominada de atenção seletiva. A atenção dividida diz respeito à capacidade que um indivíduo tem de focar dois estímulos simultaneamente.

Esta função é complexa, mas de fundamental importância para o comportamento humano e não redutível a uma simples definição ou associação com uma região específica do cérebro.

A literatura científica vem estudando os efeitos dos jogos para a cognição humana. No que diz respeito à atenção, as pesquisas têm revelado que a interação entre o homem e os jogos de videogame resulta em alterações percepto-cognitivas relevantes.

Pesquisadores como Dubar, Hill e Lewis (2001) descobriram que a habilidade de trocar o foco da atenção está voltada para o desempenho de tarefas complexas, e que quanto maior esta habilidade, melhor o desempenho. Ao investigarem a habilidade de crianças de diferentes faixas etárias em trocar o foco atencional, sua concentração e o comportamento destas crianças e seus pais no trânsito, utilizando o Frog Game, sugeriram que o comportamento destas crianças como pedestres estava relacionado com sua habilidade de prestar atenção. Constataram que as crianças que se saíram bem no jogo possuíam melhor grau de atenção e foram mais cautelosas ao atravessar a rua, e que a habilidade de trocar a atenção - por exemplo a de concentração - diferiu entre elas conforme a idade, de modo que as crianças jovens foram menos eficientes do que as mais velhas. Concluíram ainda que a troca do foco atencional e a concentração também são habilidades distintas, sendo as crianças que tinham a habilidade de trocar o foco atencional mais rapidamente no jogo de videogame mais propensas a olhar o trânsito quando estavam para cruzar uma estrada, enquanto crianças com menor habilidade de se concentrar perante um estímulo distrator tendiam a ser mais impulsivas e a cruzar a estrada de maneira menos segura. Concluíram também que existe correlação entre concentração e impulsividade, pois as crianças que responderam mais rapidamente no jogo e cometeram muitos erros obtiveram alta pontuação para impulsividade.

Green e Bavelier (2003) publicaram o resultado de uma pesquisa com uma população de jovens com idades entre 18 e 25 anos na qual os jogadores de videogame (JVGs) tinham experiência mínima de seis meses com jogos, enquanto NJVGs tinham pouca ou nenhuma experiência com jogos.

Na comparação dos desempenhos de JVGs e não NJVGs os resultados levaram às seguintes conclusões:
JVGs aumentam a capacidade do sistema de atenção visual e deveriam exaurir seus recursos de atenção visual mais lentamente em relação a NJVGs; jogar videogame melhora a atenção visual e sua distribuição espacial; JVGs foram significativamente mais acurados que NJVGs em relação à enumeração de itens; o tempo de processamento e informação visual foi menor no grupo de JVGs.

Após treinamento do grupo de NJVGs a análise dos resultados e as conclusões revelaram que o grupo de NJVGs que foi treinado no jogo de ação "Medal of Honor" teve um desempenho superior ao daquele que jogou um jogo controle ("Tretis"). Os pesquisadores concluíram então que jogar videogame de ação pode alterar significativamente o processo de atenção visual, assim como sua distribuição pelo espaço.

Castel, Pratt e Drummond (2005), utilizando um paradigma denominado de inibição do retorno conhecido como inhibition of return (IOR), que diz respeito a uma preferência atencional por locais não conhecidos e inesperados em detrimento daqueles que a pessoa já experienciou, investigaram como os jogos de videogame de ação podem influenciar a atenção seletiva visual. Estes pesquisadores examinaram semelhanças e diferenças entre JVGs e NJVGs nas habilidades de inibir a atenção para retornar a situações previamente conhecidas e de eficácia na procura visual.

Os resultados confirmaram que existem diferenças claras entre JVGs e NJVGs em tarefas visuais de atenção; contudo foram encontradas algumas semelhanças interessantes entre os dois grupos, indicativas de que processamento atencional semelhante é usado em determinadas situações. No primeiro experimento, os JVGs descobriram os alvos mais rapidamente que os NJVGs e exibiram IOR, o que indica que este efeito inibitório pode estar relacionado a um mecanismo básico de procura, de busca, que não é atenuado como consequência de se jogar videogame habitualmente. Sob estas circunstâncias a experiência com jogos de videogame teriam efeito pequeno nos mecanismos básicos envolvidos na inibição e localização da atenção visual focada. Ao contrário das conclusões de Green e Bavelier (2003), os pesquisadores sugeriram que JVGs e NJVGs contam com mecanismos semelhantes para guiar a atenção visual, porém JVGs teriam mapas mais rápidos de estímulo-resposta, que os levam a responder rapidamente na presença de alvos em um ambiente visual (Castel et al., 2005).

Nas tarefas de procura visual os JVGs apresentaram tempos de resposta mais rápidos em uma tarefa que consistia em identificar uma letra-alvo entre 
distratores tanto na tarefa mais fácil quanto na mais difícil, o que poderia ser um indicativo de que o comportamento de procura visual dos JVGs foi mais eficiente do que o dos NJVGs. Os pesquisadores também apontaram que as respostas mais rápidas dos JVGs devem ocorrer pelo fato de estes sujeitos desenvolverem fortes associações entre a presença de um estímulo e a emissão de uma resposta (Castel et al., 2005).

Para o presente trabalho é importante ressaltar que atenção visual não significa o mesmo que atenção no sentido de concentração. A atenção visual pode ser descrita de forma simples, como filtro para a percepção. Encontramos, no dia a dia, muito mais estímulos em um ambiente do que seríamos capazes de processar. Desta forma, a atenção visual é o mecanismo pelo qual alguns estímulos são selecionados para serem processados e outros, não. No geral, podemos dizer que quando estamos atentos a um determinado estímulo, seu processamento se dá de forma mais rápida e eficiente em relação àqueles estímulos que são negligenciados (Green \& Bavelier, 2004).

Assim, o objetivo deste estudo foi investigar se a performance em teste de atenção sustentada é diferente em quem joga e em quem não joga videogame e confrontar os resultados com as descobertas feitas sobre os efeitos dos jogos de videogame para aspectos relacionados com a atenção visual.

\section{MÉTODO}

\section{Participantes}

A amostra do presente estudo foi composta por 30 crianças, de classe socioeconômica baixa, com idades entre 10 e 16 anos e sete meses e escolaridade entre $5^{\mathrm{a}}$ série do Ensino Fundamental I e $2^{\circ}$ ano do Ensino Médio, que frequentavam uma organização não governamental (ONG) que atende crianças e jovens carentes em uma cidade de porte médio. A pesquisa foi realizada após a aprovação do Comitê de Ética e Pesquisa da Universidade Federal de Minas Gerais - UFMG.

Os participantes foram recrutados pela pesquisadora nas salas de aula da Instituição. Foram explicadas a natureza do trabalho e as etapas da pesquisa, assim como o cunho teórico-conceitual do trabalho (com adequação da linguagem). Uma vez feito isto, foi entregue uma folha do termo de consentimento para ser assinada pelo voluntário e/ou seus pais. (Também foi informado) Os participantes foram também informados da disponibilidade da pesquisadora para possíveis esclarecimentos.

\section{Critérios de inclusão e exclusão}

Pelo fato de essa instituição acolher algumas crianças cuja família está de passagem pela região, foram selecionadas apenas aquelas com residência fixa, para evitar possíveis perdas e garantir que a execução das etapas da referida pesquisa fosse cumprida em tempo hábil. Foram incluídas apenas crianças sem déficits visuais ou com visão corrigida (20/20), que não apresentaram deficiência mental, não tomavam medicação capaz de alterar a cognição e não apresentaram alteração no MTA-SNAP-IV (Mattos, Serra-Pinheiro, Rohde \& Pinto, 2006).

Para exclusão de deficiência mental foi aplicado o Raven Infantil (Angelini, Alves, Custódio \& Duarte, 1999) ou Raven - Escala geral (Raven, 2003), de acordo com a faixa etária. Para exclusão de déficit visual foram aplicados um teste de visão por meio de tabela de optotipo e o teste Ishihara para exclusão de daltonismo. Além do critério da residência fixa e faixa etária, foram recrutadas crianças que praticavam os jogos de videogame havia mais de seis meses (grupo controle) e crianças que nunca tiveram experiência com nenhum tipo de jogo de videogame, incluindo jogos de celular (grupo experimental). Esta pesquisa traz um diferencial em relação ao grupo de NJVGs, uma vez que esta é uma população escassa e as pesquisas relacionadas não foram tão criteriosas quanto à formação desta amostra como o foi esta investigação.

O grupo de NJVGs foi formado como uma amostra de conveniência e se constituiu de 10 crianças e adolescentes. O grupo de JVGs foi formado a partir do critério de sorteio feito por uma das professoras da instituição entre aquelas crianças que se interessaram em participar. Ao todo, participaram neste grupo 20 crianças e adolescentes.

\section{Procedimentos}

Após a definição dos grupos de JVGs e NJVGs com base nos critérios de inclusão e exclusão foi aplicado o Conners' Continuous Performance Test - CPT II (Conners' \& MHS Staff, 2006). Este teste foi aplicado em uma sala isolada de outros estímulos e de acordo com o descrito no manual. A duração da aplicação do teste foi de aproximadamente 20 minutos, aí incluída a explicação sobre o procedimento, o treinamento e sua duração. O teste foi desempenhado em um computador notebook e os sujeitos foram instruídos a apertar a barra de espaços do teclado do 
computador para todas as letras que aparecessem na tela do computador, com exceção da letra " $\mathrm{X}$ ". O tempo de apresentação do estímulo foi de 250 milissegundos (ms). Os alvos (X) e não alvos (X) foram distribuídos em seis blocos de estímulos, cada um contendo três sub-blocos com 20 tentativas cada, um para cada intervalo de apresentação do estímulo (1, 2 e 4 segundos). Os intervalos entre os estímulos são apresentados com ordem diferente em cada um dos blocos (Conners' \& MHS Staff, 2006).

Todos os adolescentes foram submetidos ao CPT. Os resultados da população de JVGs ficaram reservados para comparação. Os resultados do CPT para população de NJVGs (grupo experimental) foram considerados como linha de base para avaliação da intervenção. Os voluntários do grupo experimental foram, posteriormente, submetidos a uma exposição aos jogos "Madagascar" (Eletronic Arts, 2005) e "Harry Potter e o Cálice de Fogo" (Eletronic Arts, 2005), por 20 sessões com duração de 50 minutos/dia e uma frequência de três vezes por semana, à qual os membros do grupo de JVGs também estavam habituados. Após este período este grupo foi novamente submetido ao CPT (pós-teste).

O treinamento foi acompanhado por duas professoras da instituição previamente preparadas por meio de uma capacitação que incluiu conhecimentos na área das neurociências, aprendizagem, metodologia de pesquisa e ética, com duração de 6 horas.

\section{Conners' Continuous Performance Test (CPT)}

O CPT é um teste padrão ouro para avaliação da atenção sustentada, objeto de estudo desta pesquisa. Utilizado principalmente para diagnóstico e acompanhamento de casos de transtorno de déficit de atenção (TDAH), o teste tem sido usado para pesquisas em várias áreas, por fornecer uma rica fonte de dados (Conners' \& MHS Staff, 2006). O CPT-II requer que o sujeito se mantenha atento durante toda a tarefa que tem duração de 14 minutos.

Nesta pesquisa o instrumento foi utilizado apenas como ferramenta para acessar a habilidade em sustentar a atenção dos sujeitos, por isso foram também utilizados os parâmetros estatísticos do mesmo instrumento para análise de desempenho.

O CPT- II pode ser auditivo, visual ou verbal. Para nossa pesquisa foi adotada a forma visual do teste, que gera oito medidas para avaliação de possíveis problemas relacionados à atenção, das quais analisamos cinco.

As variáveis analisadas foram omissões, erros por ação, tempo de resposta, variabilidade e detectabilidade, as quais são descritas a seguir (Conners' \& MHS Staff, 2006).

1. A variável "omissões" corresponde ao número de alvos (não X) em que o sujeito não responde. Um grande número de omissões indica que o sujeito está literalmente não orientado e não responde ao estímulo ou apresenta uma resposta diminuída ou lenta.

2. Erros por ação correspondem ao número de vezes em que o sujeito responde na presença de " $X$ ". A velocidade de sua resposta vai afetar esta medida. De forma geral, quando tempos de reação diminuídos ocorrem com um elevado número de erros de omissão e erros por ação, existe uma evidência de desatenção.

3. Tempo de resposta significa o tempo de resposta em milissegundos para todas as tarefas em todos os seis blocos.

4. Variabilidade consiste em um método de cálculo da consistência do tempo de resposta.

5. Detectabilidade é a medida do quão bem o indivíduo discrimina entre tarefas (não-X) e não tarefas (X).

As variáveis não avaliadas foram Erro padrão do tempo de resposta (Hit-RT Std Error), que avalia consistência da velocidade da resposta e é uma medida comparativa com variabilidade, e os resultados por intervalos de estímulos, chamados de ISI (Hit Reaction time ISI Change e Hit Standard Error ISI Change), os quais, segundo as propriedades psicométricas do manual do CPT-II, não apresentaram boa correlação em situação teste-resteste.

$\mathrm{Na}$ análise estatística foram utilizados os testes não paramétricos Mann Whitney (Z) e Kruskall Wallis $(\mathrm{KW})$ e os paramétricos Teste $t(\mathrm{t})$ e Teste Exato de Fisher (F) quando as suposições de normalidade e homogeneidade foram cumpridas. A análise foi feita por meio dos softwares Minitab 15 e SPSS 15. O nível de significância adotado foi 0,05 .

As variáveis analisadas com os testes paramétricos foram: "tempo de resposta", "detectabilidade" e "erros por ação". As variáveis analisadas com testes não paramétricos foram "omissões" e "variabilidade".

\section{RESULTADOS}

A Tabela 1 compara os resultados do value do CPT-II dos JVGs com os dos NJVGs. Foram encontradas diferenças significativas para as variáveis "omissões" e "tempo de resposta". Para as demais variáveis as diferenças não foram significativas. 
Tabela 1. Comparação do Grupo de JVG X NJVG - Linha de Base (LB)

\begin{tabular}{|c|c|c|c|c|c|c|}
\hline \multirow{2}{*}{ Variáveis } & \multicolumn{2}{|c|}{ JVG } & \multicolumn{2}{|c|}{ NJVG (LB) } & \multirow[b]{2}{*}{ Estatística do teste } & \multirow[b]{2}{*}{ p valor } \\
\hline & Média & Dp & Média & Dp & & \\
\hline Omissões\% & 1,70 & 1,64 & 5,20 & 4,45 & $K W=2,66$ & $0,008 *$ \\
\hline Erros por ação\% & 56,94 & 20,64 & 55,52 & 25,65 & $t=-0,15$ & 0,870 \\
\hline Tempo de resposta & 372,30 & 54,86 & 410,75 & 39,55 & $\mathrm{t}=1,72$ & $0,048^{*}$ \\
\hline Variabilidade & 10,20 & 5,66 & 14,31 & 9,24 & $\mathrm{KW}=1,23$ & 0,218 \\
\hline Detectabilidade & 0,46 & 0,27 & 0,51 & 0,46 & $\mathrm{Z}=0,350$ & 0,726 \\
\hline
\end{tabular}

* significativo $\mathrm{p}<0,05$

$\mathrm{Na}$ tabela 2 os resultados do value do CPT-II revelaram que após o regime de treinamento instituído o grupo de NJVGs obteve uma melhora significativa nas variáveis analisadas (omissões, erros por ação, tempo de resposta, variabilidade e detectabilidade).

Tabela 2. Comparação dos Resultados do Grupo de NJVG (LB) e Após a Intevenção (AI)

\begin{tabular}{|c|c|c|c|c|c|c|}
\hline \multirow[t]{2}{*}{ Variáveis } & \multicolumn{2}{|c|}{ NJVG $($ LB $)$} & \multicolumn{2}{|c|}{ NJVG (AI) } & \multirow{2}{*}{$\begin{array}{c}\text { Estatística } \\
\text { do teste }\end{array}$} & \multirow[b]{2}{*}{ p valor } \\
\hline & Média & dp & média & dp & & \\
\hline Omissões\% & 5,20 & 4,45 & 2,07 & 2,43 & $\mathrm{KW}=1,97$ & $0,049 *$ \\
\hline Erros por ação\% & 55,52 & 25,65 & 39,27 & 22,07 & $\mathrm{t}=3,09$ & $0,020 *$ \\
\hline Tempo de resposta & 410,75 & 39,55 & 366,71 & 53,02 & $\mathrm{t}=2,43$ & $0,015^{*}$ \\
\hline Variabilidade & 14,31 & 9,24 & 6,79 & 6,12 & $\mathrm{KW}=2,49$ & $0,013 *$ \\
\hline Detectabilidade & 0,51 & 0,46 & 0,78 & 0,41 & $\mathrm{t}=2,44$ & $0,014 *$ \\
\hline
\end{tabular}

* significativo $\mathrm{p}<0,05$

A tabela 3 apresenta a análise dos resultados entre os grupos de JVGs e NJVGs após a intervenção.

Tabela 3. Comparação do Grupo de JVG X NJVG (AI)

\begin{tabular}{lccccccc}
\hline \multicolumn{1}{c}{ Variáveis } & \multicolumn{2}{c}{ JVG } & \multicolumn{2}{c}{ NJVG (AI) } & \multicolumn{2}{c}{ Estatística } \\
\cline { 2 - 5 } & média & dp & Média & Dp & & do teste & P valor \\
\hline Omissões\% & 1,70 & 1,64 & 2,07 & 2,43 & & $\mathrm{KW}=0,31$ & 0,757 \\
Erros por ação\% & 56,94 & 20,64 & 39,27 & 22,07 & & $\mathrm{t}=-2,11$ & $0,039^{*}$ \\
Tempo de resposta & 372,30 & 54,86 & 366,71 & 53,02 & $\mathrm{t}=-0,27$ & 0,792 \\
Variabilidade & 10,20 & 5,66 & 6,79 & 6,12 & $\mathrm{KW}=2,38$ & $0,017^{*}$ \\
Detectabilidade & 0,46 & 0,27 & 0,78 & 0,41 & $\mathrm{t}=2,532$ & $0,017^{*}$ \\
\hline
\end{tabular}

* significativo $\mathrm{p}<0,05$

Observou-se que "omissões" e "tempo de resposta" não foram significativamente diferentes em relação ao grupo de JVGs. Para as demais variáveis verificou-se que o grupo de NJVGs pós-intervenção obteve uma diferença estatisticamente significativa para "erros por ação", "detectabilidade" e "variabilidade", apresentando uma melhor performance que o grupo de JVGs

\section{DISCUSSÃO}

Um melhor tempo de resposta dos JVGs em relação aos NJVGs está bem descrito na literatura especializada (Castel et al., 2005; Subrahmanyan, Greenfield, Kraut \& Gross, 2001; Green \& Bavelier, 2004; Dye, Green \& Bavelier, 2009). Em relação à variável "omissões", o melhor desempenho dos JVGs em relação aos NJVGs é igualmente descrito na literatura. Também Green e Bavelier (2003), ao aplicarem um teste em que os sujeitos deveriam identificar corretamente um " $X$ ", verificaram que os JVGs se saíram melhor do que os NJVGs no número de detecções corretas. A melhora dos tempos de resposta se deve a uma melhor coordenação mãoolhos e ao fato de o tempo de processamento da informação visual ser menor em JVGs. 
Um melhor desempenho do grupo de NJVGs após o treinamento também está bem descrito na literatura. É importante frisar que o "tempo de resposta" influencia o número de "omissões" e "erros por ação"; ou seja, o grupo de NJVGs, após a intervenção, melhorou seus tempos de resposta, apresentou-se mais orientado e respondeu ao estímulo-alvo um maior número de vezes, cometeu menos erros por ação, melhorou a consistência da velocidade da resposta e sua capacidade de discriminar entre alvo e não alvo. Isto se deve ao aumento da acurácia das respostas emitidas por estes jogadores, uma vez que aumenta a capacidade de seu sistema de atenção visual, portanto seus recursos de atenção visual se exaurem mais lentamente.

Dye, Green e Bavelier (2009) apontam que, em seu estudo, JVGs responderam mais rapidamente à tarefa do que NJVGs, mas nem por isso cometeram mais erros do que estes últimos. Este melhor processamento da informação visual sem prejuízo da exatidão da resposta tem sido bem descrito pela literatura.

Constitui uma limitação dos resultados o fato de não ter sido possível controlar o tempo e a frequência da exposição anterior dos integrantes do grupo de JVGs aos jogos de videogame utilizados nesta pesquisa, embora se tenha garantido que nos seis meses precedentes à pesquisa todos os componentes deste grupo foram expostos aos jogos. Outra limitação foi o não controle da habilidade dos participantes em jogar, uma vez que é usual na instituição eles se sentarem em duplas e jogarem com um único personagem utilizando a manete do jogo e os comandos do teclado. A alternância em determinados momentos para compartilhar da atividade pode suprir uma eventual dificuldade do colega.

Um fator importante, mas não controlado por escala padronizada, foi a sudorese nas mãos observada no grupo de JVGs durante o teste. Este fenômeno pode ser indicativo de ansiedade e esta pode ser fator interveniente em desempenhos. Por outro lado, temos também o fator motivacional do grupo de NJVGs. Um aspecto importante a ser considerado para este grupo foi ter-se percebido que o grupo dos meninos estava menos motivado para os jogos escolhidos do que o das meninas. A preferência dos meninos do grupo de NJVGs era pelo jogo "Need for Speed", o qual não é contemplado para a intervenção e é o preferido pelas crianças do sexo masculino na instituição.

Ainda que os achados desta pesquisa apontem melhor desempenho do grupo de JVGs neste teste, salientamos que esta investigação tem como um de seus principais objetivos instigar pesquisas com este paradigma em nosso país, uma vez que não temos know-how em pesquisas que envolvem videogames. Só assim poderemos alicerçar a opinião científica brasileira a respeito dos jogos e suas consequências para a cognição, bem como buscar o desenvolvimento de novas tecnologias baseadas nestes achados cuja aplicação possa ser de interesse para a reabilitação cognitiva.

É surpreendente a quantidade de habilidades cognitivas modificadas, incluindo a melhora significativa da coordenação mãos-olhos, das habilidades motoras finas, da atenção visual e da percepção de profundidade, a partir da interação com os videogames e do grau em que estas modificações ocorrem. Não obstante, é de fundamental importância que, ao analisarmos diferentes pesquisas envolvendo jogos, saibamos o que cada uma considera como JVG e NJVG, para podermos interpretar adequadamente os resultados destes estudos.

Pesquisas na área apontam os jogos de ação como aqueles capazes de levar a modificações cognitivas mais significativas do que jogos de aventura (Green \& Bavelier, 2003, 2004, 2006, 2009). Green e Bavelier (2009) ressaltam que os jogos de videogame do gênero ação melhoram os recursos atencionais de jogadores de qualquer idade.

\section{CONCLUSÃO}

Estudos recentes vêm demonstrando que crianças e adolescentes envolvidos com jogos de videogame apresentam maior habilidade para alocar de forma mais eficiente seus recursos atencionais tanto no que diz respeito ao espaço quanto ao tempo. A análise dos resultados corrobora estes achados e demonstrou que os jogos de videogame foram eficientes para melhorar o desempenho destes adolescentes no teste de atenção sustentada CPT-II em - relação às variáveis relacionadas com a atenção.

Salientamos a necessidade de novos estudos para melhor elucidar os efeitos do videogame para cognição e do desenvolvimento de novas tecnologias baseadas nestes achados cuja aplicação possa ser útil à reabilitação cognitiva.

\section{REFERÊNCIAS}

Angelini, A. L., Alves I. C. B., Custódio, E. M. \& Duarte, J. L. M. (1999). Matrizes Progressivas Coloridas de Raven. São Paulo: Centro Editor de Testes e Pesquisas em Psicologia. 
Castel, A. D., Pratt, J. \& Drummond, E. (2005). The effects of action video game experience on time course of inhibition of return and efficiency of visual search. Acta Psychologica, 119, 217-30.

Conners' K. C. \& MHS Staff (2006). Conner's Continuous Performance Test (CPT II) - Technical Guide and Software Manual. Canada: MHS.

Dubar, G., Hill, R. \& Lewis, V (2001). Children's attentional skill's and road behavior. Journal of Experimental Psychology: Applied, 7(3), 227-34.

Entertainment Software Association - ESA. Essential facts about the computer and videogame industry. ESA Recuperado em 12 de novembro de 2007 em http://www.theesa.com/facts/pdfs/ESA_EF_2006.pdf

Green, C. S. \& Bavelier, D. (2003). Action Video game modifies visual selective attention. Nature, 423, 534-37.

Green, C. S. \& Bavelier D. (2004). The Cognitive Neuroscience of Video Games. In Messaris P., Humphreys L., (Eds.) Digital Media: Transformations in Human Communication (pp. 211-224). New York: Peter Lang.

Green, C. S. \& Bavelier, D (2006). Enumeration versus multiple object tracking: the case of action video game players. Cognition, 101, 217-45.
Dye, M. W. G., Green, C. S. \& Bavelier, D. (2009). The development of attention skills in action video game players. Neuropsychologia, 47, 1780-89.

Mattos, P., Serra-Pinheiro, M. A., Rohde, L. A. \& Pinto, D. (2006). Apresentação de uma versão em português para uso no Brasil do instrumento MTA-SNAP-IV de avaliação de sintomas de transtorno de déficit de atenção/hiperatividade e sintomas de transtorno desafiador e de oposição. Rev. Psiquiatr. Rio Gd. Sul, 28(3), 290-97.

Posner, M. I. \& Fan, J. (2006). Attention as an organ system. Sackler Institute Recuperado em 5 de abril de 2008 em http://www.sacklerinstitute.org/users/jin.fan/publications/ ANT_AS_ORGAN_SYSTEM.pdf

Raven J. C. (2003). Teste das Matrizes Progressivas Escala Geral. Rio de Janeiro: CEPA.

Subrahmanyan, K., Greenfield, P., Kraut, R. \& Gross, E. (2001). The impact of computer use on children's and adolescents' development. Applied Developmental Psycholog, 22, 7-30.

Recebido em 27/08/2009 Aceito em 20/05/2010

\section{Endereço para correspondência:} Luciana Alves. Rua São Paulo, 351/1403, Centro, CEP 30170-130, Belo Horizonte-MG, Brasil. E-mail: lualbhmg@gmail.com. 